\title{
Discourse formulation and neurovascular activation across four genres
}

\author{
Michael S. Cannizzaro' ${ }^{1}$ Shaun R. Stephens ${ }^{2}$ \\ ${ }^{1}$ Department of Communication Sciences and Disorders, University of Vermont, Burlington, $V_{i}{ }^{2}$ Vermont Center for Clinical and Translational \\ Science, University of Vermont, Burlington, T, USA
}

\begin{abstract}
Purpose: Discourse production is a naturalistic and commonly occurring form of communication. Thus, it is no surprise that discourse formulation is a clinically relevant assessment for persons who experience communication impairments associated with cognitive disruptions such as traumatic brain injury, mild cognitive impairment, and dementia. The most commonly studied genre of discourse is the production of fictional narratives; however, little is known about the formulation of other, more commonly occurring and ecologically valid forms of discourse. To better understand the communication behaviors associated with these other discourse forms, this pilot research examined efficiency, informativeness, and neurovascular activation during formulation of procedural, personal, conversation, and fictional discourse produced by neurologically healthy adults.
\end{abstract}

Methods: Ten healthy adults formulated spoken discourse in four clinically important genres. Efficiency and informativeness characteristics were analyzed, and prefrontal neurovascular activation was measured using functional near infrared spectroscopy.

Results: Efficiency was similar between the four genres. Informativeness varied significantly by genre and was lowest in complex procedural discourse and highest in conversation. Differences in location and magnitude of neurovascular activation between genres were not significant. Significant negative relationships and trends between discourse efficiency and neurovascular activation were observed, suggesting a negative relationship between discourse performance and neurovascular processing demands.

Conclusions: Measurement of discourse efficiency enables meaningful comparison between four genres of discourse. Therefore, the measurement and assessment of functionally relevant genres of discourse is feasible, and may be more relevant than fictional storytelling for individuals with cognitive-communication impairments.

Keywords: Discourse genre, Narrative language, Functional near infrared spectroscopy (fNIRS), Neurovascular activation, Macrostructure, Microstructure

\section{INTRODUCTION}

Communication through discourse is the central method of human interaction. Discourse is a series of interconnected propositions that communicate a coherent message that is above the level of single sentences $[1,2]$. Formulating and understanding discourse is a linguistically and cognitively demanding task, and the ability to efficiently produce coherent discourse is critical for functional participation in family and social relationships, vocation, and community engagement [3-6]. Discourse analysis has

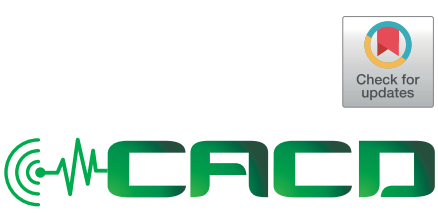

Received: February 2, 2019

Revision: March 30, 2019

Accepted: April 3, 2019

\section{Correspondence:}

Michael S. Cannizzaro

Department of Communication Sciences and Disorders, University of Vermont, 489 Main St, Burlington, VT 05405-1709, USA

Tel: +1-802-656-9725

Fax:+1-802-656-2528

E-mail: michael.cannizzaro@med.uvm.edu

(C) 2019 The Korean Association of SpeechLanguage Pathologists

This is an Open Access article distributed under the terms of the Creative Commons Attribution NonCommercial License (http://creativecommons.org/ licenses/by-nc/4.0/) which permits unrestricted noncommercial use, distribution, and reproduction in any medium, provided the original work is properly cited. 
proven a useful tool for quantifying discourse formulation and comprehension in individuals with cognitive-communication disorders following traumatic brain injury (TBI) [7-10], in children with language impairments [11], in neuropathologies of aging [12-14], and in normal aging [15,16]. Individuals with closed head injury TBI commonly have difficulty with macrostructural and some microstructural aspects of discourse even in the absence of overt aphasia [3,17-19]. These discourse disruptions can occur in individuals with TBI of any severity, including mild (also called "concussion") [10,18,2023]. Persistent impairments in discourse have been associated with a loss of independence, social isolation, lack of community reintegration, decline in vocational position, depression, and lower quality of life [5,24].

Discourse analysis is typically subcategorized into microlinguistic elements (within sentence measures) and macrostructural features (relations between sentences and the overall theme of the discourse). Microlinguistic elements include productivity, speed or fluency, grammaticality and grammatical complexity, and cohesion (inter-sentential relationships at the local level, without explicit reference to the narrative theme) $[7,10,17,19,25-29]$.

Macrostructural features include coherence (the relevance of utterances to the theme of the discourse), informativeness (also called completeness), organization (story structure or story grammar), content accuracy (also called critical content), topic management; efficiency (combining critical content and speed), and quality $[1,4,12,17,19,21,30-33]$. Lê and colleagues [4] used a combination of completeness and story grammar to derive a story quality indicator, "story goodness;" they argued plausibly that this should be considered an important metric, as it is what is typically measured in functionally relevant settings: academic settings for children and adolescents, and vocational settings for adults; see also [34]. Pistono et al. [15] categorized discourse formulation into four "clusters of informativeness" among older adults without neuropathology or cognitive impairment, based on their use of story grammar, coherence markers, and production of tangential information that impaired informativeness.

Discourse is typically categorized into various genres, based on the function that the discourse serves: procedural narratives, personal narratives, conversation, exposition, and fictional narrative, for example. In formulating effective discourse in each genre, the speaker must incorporate knowledge of the topic, the context, the communication partner's prior knowledge, and ancillary variables such as time con- straints. Most studies of discourse in individuals with TBI over the past 15 years have focused on examining the genre of fictional narratives: most frequently picture description, wordless picture books, or story retelling [12,33,35]. In functional natural communication, however, monologic fictional storytelling is an infrequent genre $[19,36]$. Among children it has been explicitly demonstrated to be a non-preferred discourse modality [34,37], and researchers have been exhorted to explore more functional genres of discourse. There remain, however, very few published investigations examining discourse in genres other than fictional. Whitworth and colleagues [16] characterized the discourse of 30 healthy adults from three age bands (20-39, 40-59, 60+ years old) in four genres: recount (personal narrative), procedural, exposition (opinion), and narrative (retelling a fictional narrative) and found coherence to be consistent between the age groups, but informativeness to be slightly lower with older participants. The Whitworth et al. study notwithstanding, research on socially relevant (functional) genres of discourse is sparse.

The goal of this research is therefore to measure discourse formulation in three ecologically relevant genres of discourse in addition to fictional storytelling. We measured one microlinguistic and one macrolinguistic characteristic as well as neurovascular activation during formulation of procedural, personal, conversation, and fictional discourse by adults without brain injury. This current study design is being conducted to explore measurement across meaningful discourse genres, to serve as a feasibility study future investigations that will include persons with cognitive-communication disorders and to explore the relationships between behavioral discourse demands and demands on the prefrontal cortex.

Efficiency (the microlinguistic measure) and informativeness (the macrolinguistic measure) were analyzed for all four genres. These two measures were chosen because they have utility across genres and therefore are not tied to a particular genre or context [19], and because they have been reported to be measurably impaired in individuals with mild TBI [21], as well as in individuals with moderate or severe TBI. Approximately $80 \%$ of brain injuries are classified as 'mild', with Glascow Coma Score at time of injury >13 [38], and approximately $50 \%$ of those injuries result in persistent post-traumatic symptoms [39], so it is of particular interest to explore aspects of commonly occurring forms of discourse that are impaired among individuals with mild to moderate TBI. The discourse features that are impaired in individuals with mTBI are typically also impaired in individuals with moderate and severe 
TBI, and they therefore to have particular clinical relevance for speech-language pathologists working with adult clinical populations. These two measures also carry information about speed, productivity, thematic relevance, content accuracy, and informativeness in two concise numbers. Discourse efficiency has also been posited as a driving force in the development of human language [40], and disruptions in efficiency are common complaints among individuals with non-aphasic language impairment and their families [8]. Story grammar was not included despite prevalence of this measure in the research literature because it applies primarily to fictional narratives, and not to other, more ecologically important forms of discourse (e.g., procedural, conversation).

Research approaches which unify communication behavioral performance and functional neuroimaging have improved our understanding of component-specific neural networks that support discourse processing and formulation [4143]. During discourse comprehension, increasing neural activity and spreading activation have been observed throughout the extended language network (ELN) [44], including the posterior cingulate cortex, inferior precuneus (Brodmann's area BA23/31) and aspects of the dorso-medial prefrontal cortex (BA 9/10). Xu et al. [45] observed similar activation patterns during story narrative processing: bilateral activity in the prefrontal regions (BA 8, 9, and 10) which increased as language tasks required higher levels of integration (i.e., words $<$ sentences $<$ narratives). These prefrontal regions also respond differentially based on the magnitude of task demands, and adherence to a predictable narrative structure lowers task demands: during the reading of structured narratives, the organizational structure of the narrative reduces processing demands on the prefrontal cortex (PFC) compared to the reading of similar length unconnected sentences [46]. Although most research has focused on discourse comprehension (rather than formulation), there is evidence of considerable overlap between the neural architecture used for processing and formulating discourse: extrasylvian areas that interact with the language system during both production and comprehension of discourse (i.e., dorsomedial prefrontal cortex, precuneus and inferior parietal lobules, presupplementary motor area and left dorsal premotor cortex) [47].

Until recently, functional neuroimaging during discourse formulation has been difficult or impossible because of head motion artifacts introduced into the imaging data during speaking, and because equipment-related constraints made naturalistic communication impossible, for example the loud noise, confined space, and movement restrictions in functional magnetic resonance imaging (fMRI). This has effectivly limited the ecological validity of discourse research [48]: most research on the neural architecture underlying discourse has been derived from studies of receptive language in the form of silent text reading, "covert" articulation, or thinking about overlearned and rehearsed stories while lying silently in a scanner. Some studies have used fMRI combined with positron emission tomography (PET) or continuous arterial spin labeling (CASL) to allow imaging during overt speech [33,47], but these studies remain physically restrictive and costly, limiting ecological applicability. In the past five years, however, functional near infrared spectroscopy (fNIRS) has seen increasing use in language studies. fNIRS is an optical neuroimaging modality that avoids many of the more problematic issues with other neuroimaging methods: it is robust to the effects of motion artifacts from head and muscle movements, is silent, and is not physically restrictive [48]. These attributes make fNIRS ideal for studying brain activation patterns during formulation of functional discourse in ecologically relevant contexts.

Given the lack of research on formulation of functionally relevant genres of discourse, and the prevalence of disrupted discourse among individuals with TBI, this exploratory pilot study sought to compare naturalistic discourse formulation within individuals across four different genres. Outcome measures were behavioral performance (efficiency, informativeness) and neurovascular activation amplitudes. The study was designed to test four hypotheses:

1. There will be no significant differences in efficiency between genres.

2. There will be significant differences in informativeness between genres.

3. There will be significant differences in the magnitude of neurovascular activation between genres.

4. Discourse with higher efficiency will have lower neurovascular activation.

\section{METHODS}

\section{Participants}

Subjects were recruited from flyers and e-mail distribution lists at the university. Participants were excluded if they had conditions that have been reported to result in discourse impairments: prior traumatic brain injury, schizophrenia, neurological impairment, autism or developmental disability. 
Table 1. Demographic characteristics of participant group

\begin{tabular}{lccc}
\hline & Mean & $S D$ & Range \\
\hline Age & 20.6 & 1.26 & $18-22$ \\
Education (year) & 14.6 & 0.97 & $13-16$ \\
Gender female (male) & $9(1)$ & & \\
Handedness R (L) & $9(1)$ & & \\
\hline
\end{tabular}

Sample size calculations were designed to detect a difference in means on the efficiency measure (CIUs per minute) between participants without TBI, using pilot data from the Matsuoka et al. study [28], in table 3 of that article: a difference in means between 0.93 (0.3 SD, normal controls) and 0.62 (0.2 $S D$, TBI group). Calculations were performed using a twosided t test with two samples to achieve 0.80 power at $\alpha=0.05$ significance level, assuming unequal variances. This yielded a target sample size of 12 in the non-brain injured group. Eighteen subjects were recruited; four were excluded because further discussion revealed that they had had prior TBI; fNIRS data were lost or corrupted for three subjects; one was subsequently removed as an outlier on the efficiency measure, yielding 10 participants. Demographic variables are presented in Table 1. The study was approved by the University Institutional Review Board, and informed consent was obtained for each participant. Subjects were tested individually in a quiet room in the laboratory.

\section{Experimental design}

Baseline expressive language tasks (counting and formulation of single sentences) were used to differentiate discourse-level neurovascular activation from sentence-level activation. Participants were requested to count aloud from 1-100, and to formulate single sentences. Two different stimuli were used to elicit baseline single sentences, one textual and one visual: the textual stimulus used the "Sentence Assembly" task from the Clinical Evaluation of Language Fundamentals 5th Edition (CELF-5). The visual stimulus was a set of black and white line drawings of action verbs from the International Picture Naming Project (IPNP) [49]. Participants were instructed “On each of the following slides you will see 6 pictures of actions. Use each picture to create a sentence that describes the picture." Each stimulus method (textual and visual) was used to elicit formulation of unconnected single sentences for a period of 60 seconds.

To elicit procedural discourse, participants were given two tasks: the "ATM" task: “Tell me all the steps involved in with- drawing money from an ATM, as if I had never done it before" [50], and the "NYC" task: "Imagine that you are going on a vacation a week from now. You are traveling to New York City for a two-week stay. Think about all you will have to do to get ready to go, such as how you will get there, what you will bring, and what you will do," described in Fleming and Harris [51]. Participants were allowed as much time as needed to complete the task.

Personal narratives were elicited with the conversational map procedure, as described in McCabe et al. [34] and Peterson and McCabe [52]: a verbal prompt was given that briefly described two personal experiences of the elicitor: going on summer vacation, and a family member getting injured. Both experiences were elicited in order to compare discourse from experiences with positive (vacation) versus negative (injury) valences: the participant was asked to tell about two such episodes in their life. Neutral cues were used to encourage elaboration where appropriate ("and?" "anything else?"), to support discourse while not influencing the content of the message.

The elicitation procedure for the fictional narrative was described in Berman and Slobin [53]. We presented a color picture of the painting "The Runaway" by Norman Rockwell. Participants were instructed as follows: "Tell me a story about the picture below. The scene in this picture represents a moment in time. Something happened to cause the pictured event, and something is going to happen afterwards. When the slide changes, please tell me the whole story from what happened before the pictured event through what will happen after this scene." Participants were given as much time as needed to complete the task. Conversation was elicited as described in Snow, Douglas, \& Ponsford [50]: the examiner explained "Now you're going to have a short conversation with the examiner," and the examiner asked about the participant's family, what kind of work the participant does, and what they like to do on the weekend. A schematic illustration of the overall experimental structure is presented in Figure 1.

\section{Narrative content analysis}

All discourse was transcribed verbatim by two trained students in the Communication Sciences and Disorders BS program at UVM: there were a total of 60 discourse transcripts (6 per participant: 2 procedural, 2 personal, 1 fictional, 1 conversation). Inter-rater reliability was assessed through random selection and reanalysis of $10 \%$ of scores by the first author and $30 \%$ of scores by the second author; inter-rater reliability was $>90 \%$. Data were analyzed and results presented graphi- 


\begin{tabular}{|c|c|c|c|c|c|c|c|c|}
\hline \multicolumn{3}{|c|}{ Baseline Measures } & \multicolumn{2}{|c|}{ Procedural Discourse } & \multicolumn{2}{|c|}{ Personal Narrative } & Fictional & Conversation \\
\hline $\begin{array}{c}\text { Count } \\
1-50\end{array}$ & $\begin{array}{c}\text { Sentence } \\
\text { Assembly } \\
(60 \mathrm{~s})\end{array}$ & $\begin{array}{c}\text { Picture } \\
\text { Descript } \\
(60 \mathrm{~s})\end{array}$ & $\begin{array}{l}\text { NYC Trip } \\
(60 \mathrm{~s})\end{array}$ & $\begin{array}{c}\text { ATM } \\
\text { Machine } \\
(60 \mathrm{~s})\end{array}$ & $\begin{array}{l}\text { Injury } \\
(60 \mathrm{~s})\end{array}$ & $\begin{array}{c}\text { Vacation } \\
(60 \mathrm{~s})\end{array}$ & $\begin{array}{c}\text { Runaway } \\
(60 \mathrm{~s})\end{array}$ & $\begin{array}{c}\text { Conver- } \\
\text { sation w } \\
\text { Examiner } \\
(60 \mathrm{~s})\end{array}$ \\
\hline
\end{tabular}

Figure 1. Schematic of the study design. Gray bar represents instructions to participants; white bar is 20 second fixation cross between tasks.

cally using Stata version 15.1, except for Hotelling's t-squared tests comparing behavioral outcomes, which were carried out using SPSS. Efficiency was analyzed using the number of correct information units (CIUs) per minute, as described in Nicholas and Brookshire [29] and Matsuoka, Kotani, and Yamasato [28]. Detailed information on CIU analysis is in Nicholas \& Brookshire [29] (Appendix B of that article): in summary, all incorrect words (such as fillers, inaccurate words, mazes, pronouns without referents, commentary on the task) were removed from the word count, and the resulting CIUs are divided by duration of the spoken utterance in minutes to yield CIUs/minute. This measure has the advantage of presenting information about speed, grammatical accuracy and content accuracy in a single score.

Informativeness was analyzed using the task relevant method for each of the six measurements: Procedural_ATM, Procedural_NYC, Personal_Vacation, Personal_Injury, Fictional, Conversation. There are established protocols for analyzing each of these six measures: Procedural_ATM was measured as a Percent of Essential Steps, as described in [50]: there are considered to be eight "essential" steps in withdrawing money from an ATM, and the number of steps described by the individual is recorded, with the number of steps described divided by the total essential steps yielding a percentage. Procedural_NYC was analyzed using the Core Elements procedure [51], which is a $0-26$ point checklist: the number of items described by the individual was divided by the total 26 possible steps, yielding a percentage.

Personal_Vacation and Personal_Injury were analyzed using the sum of High Point Analysis (0-7 point rating scale) [52] and the narrative scoring rubric of the Massachusetts Comprehensive Assessment System (MCAS), a 0-6 point scale described in [34]. High Point Analysis and the MCAS were combined because unlike High Point Analysis, the MCAS includes indicators of discourse quality, which in most educational and vocational contexts is more functionally relevant than adherence to a conventional narrative structure. It includes for example ratings for "Rich topic/idea development, Careful and/or subtle organization, Effective/rich use of language." High Point Analysis analyzes discourse by clause for the presence of seven constructs in a narrative: 1 . opener; 2 . orientation/description; 3. complicating action; 4. climax (i.e., "high point"); 5. resolution; 6. evaluation; 7. closure. The sum of High Point Analysis (6 point scale) and MCAS (7 point scale) yielded a 0-13 point scale. Each individual's score was divided by the total 13 possible to yield a percentage.

Fictional narratives were scored using the Narrative Scoring Scheme (NSS) rubric [31], which measures narrative macrostructure in seven domains: three story grammar components (Introduction, conflict resolution, conclusion) and four story "quality" markers (character development, mental states, clarity of referencing, cohesion). Each of these seven domains is measured along a 5-point continuum, yielding a 35 point total; the individual's score was divided by the total 35 points possible, yielding a percentage. Conversation was analyzed using a modification of the Clinical Discourse Analysis tool [50,54], a conversational sampling procedure providing a descriptive analysis of functional aspects of social interaction. The CDA measures discourse in four domains, following the "cooperative principle" of Grice [55], gauging communication according to quantity, accuracy, relation (relevance), and manner. Within these four domains there are 17 possible "problem behaviors:" for example, within Quantity, discourse is analyzed for "Insufficient information, Nonspecific vocabulary, Informational redundancy, Need for repetition." Discourse samples were transcribed and segmented into t-units: "minimally terminable units," as described in $[1,56]$. Each utterance was gauged as to whether it contained an error in any of the domains (errors in two or more different areas were not counted twice: an utterance was simply gauged as having an error or not). The number of error-free t-units was divided by the total number of t-units in the sample, yielding a percentage. For both efficiency and informativeness, paired-samples Hotelling's $t$-squared test $\left(t^{2}\right)$ was used to compare differences in means between the genres, because of the multivariate nature of the sample (more than one parameter for each sample). 


\section{fNIRS instrumentation and signal processing}

Neurovascular activation was measured using a 16-channel continuous wave fNIRS system (Biopac fNIRS 100, Biopac Systems, Goleta, CA) to monitor changes in the concentration of oxygenated $\left(\mathrm{HbO}_{2}\right)$ and deoxygenated $(\mathrm{Hb})$ blood flow in the PFC relative to an established stable baseline. The fNIRS device uses 16 optodes (optical sensors) positioned over the frontal cortex to measure changes in optical density in cerebral bloodflow during task-related activity. The fNIRS headband was placed on participants according to optimal positioning guidelines following the manufacturer's instructions: using the international 10-20 system for standardized electrode placement in electroencephalography, the lower row of channels was placed on the forehead so the horizontal ( $\mathrm{x}$ ) axis coincided with the symmetry axis of the head (i.e. between the eyes). On the vertical (z) axis, the sensor was positioned at Fpz, with Fp1 and Fp2 marker locations positioned at the bottom voxel row [57]. The lower row of channels is then aligned with Fp1, Fpz and Fp2. The detectors cover PFC areas BA 44, 45,9 , and 10 [58]. Soft foam tape was used to cover the outer surface of the device and prevent the intrusion of ambient light to the detectors. Participants were asked to refrain from large head movements or forehead movements. Activation was measured during the first 60 seconds of each task, and recorded as average oxyhemoglobin $\left(\mathrm{HbO}_{2}\right)$ levels for each optode. These values were transformed to beta-weights to eliminate nonsignificant activations (i.e. activations which were not significantly greater than the baseline counting condition).

$\mathrm{HbO}_{2}$ data was derived from the 16 optodes at a sampling rate of $2 \mathrm{~Hz}$. All recordings were made following a short device testing period to ensure adequate signal characteristics for the OD data (i.e. registered steady-state values between 800 and 4,000 mV during a no-task condition). All fNIRS optical density signal data were captured and recorded through the Cognitive Optical Brain Imaging Studio software package [58]. Real-time monitoring of the raw signal and calculated percent change signal values was used to detect motion artifacts and potential loss or oversaturation of signal due to poor headband contact with the participant's forehead. Data analysis was conducted using Statistical Parametric Mapping for Near Infrared Spectroscopy software package (NIRS-SPM Version 4.1) [59] according to manual specifications [59]. Data were inspected in the NIRS-SPM time-series window for signal integrity and correspondence to the experimental design timeline.

To remove signal contamination related to nonrelevant biological signals (e.g., scalp bloodflow, cardiac and systemic bloodflow, vasomotion), wavelet minimum description length (wavelet-MDL) detrending was applied to each data set: raw signal is decomposed into various subcomponents at distinct scales to isolate the clinically relevant signal [60,61]. To correct for serial correlations in the data, precoloring of the data set was used with low-pass filter, hemodynamic response function shape, and full width at half maximum $=4$ seconds [59]. Individual activation maps and significant $\beta$-weights are all reported with significance levels set at $p<0.05$, using Lipschitz-Killing curvature with Euler characteristics to account for expected correlations [59].

Significant $\beta$-weight activations for each participant were then aggregated into four regions of interest (ROI): left lateral, left medial, right medial, and right lateral. This facilitated comparison of neurovascular activation between genres across participants. Analysis of neurovascular activation during Procedural discourse was narrowed to just the NYC task, because preliminary results indicated significant ceiling effects for the ATM task, and the NYC task was gauged to be a more realistic proxy for real-world moderate complexity planning. Among Personal narratives the Injury task (rather than the Vacation task) was selected for comparison with neurovascular activation. Correlations between behavioral data and neurovascular activation were explored using pairwise Pearson product-moment coefficients.

\section{RESULTS}

The characteristics of discourse efficiency and informativeness are presented in Table 2. A paired-samples Hotelling $t$ squared test $\left(t^{2}\right)$ was used to compare differences in means between the genres, as noted in Table 2.5. Multiple comparison correction (Bonferroni) was not applied, following recommendations in Perneger [62], because of its assumption of a universal null hypothesis and because of the risk of such

Table 2. Behavioral performance on discourse formulation tasks

\begin{tabular}{lcc}
\hline & $\begin{array}{c}\text { Efficiency in } \\
\text { CIU/min mean }(S D)\end{array}$ & $\begin{array}{c}\text { Informativeness } \\
\% \text { mean }(S D)\end{array}$ \\
\hline Procedural: ATM & $148(28)$ & $76.3(16.1)$ \\
Procedural: NYC & $133(37)$ & $29.2(7.3)$ \\
Personal: Vaca & $129(19)$ & $62.9(7.4)$ \\
Personal: Injury & $129(20)$ & $68.6(9.0)$ \\
Fictional & $132(24)$ & $61.1(10.8)$ \\
Conversation & $112(15)$ & $80.3(9.0)$ \\
\hline
\end{tabular}

CIU, correct information units. 
Table 2.5. Paired samples tests: Informativeness by genre

\begin{tabular}{lccccc}
\hline & NYC $(t, p)$ & Vaca $(t, p)$ & Inj $(t, p)$ & Fict $(t, p)$ & Conv $(t, p)$ \\
\hline ATM & $8.065,<0.001^{*}$ & $2.931,0.017^{*}$ & $1.100,0.300$ & $2.253,0.051$ & $-0.745,0.475$ \\
NYC & - & $-9.494,<0.001^{*}$ & $-9.545,<0.001^{*}$ & $-8.332,<0.001^{*}$ & $-17.299,<0.001^{*}$ \\
Vaca & - & - & $-1.809,0.104$ & $0.350,0.735$ & $-6.400,<0.001^{*}$ \\
Injury & - & - & - & $1.372,0.203$ & $-3.656,0.005^{*}$ \\
Fict & - & - & - & - & $-3.650,0.005^{*}$ \\
Conv & - & - & - & - & - \\
\hline
\end{tabular}

*Significant at $p \leq 0.05$.
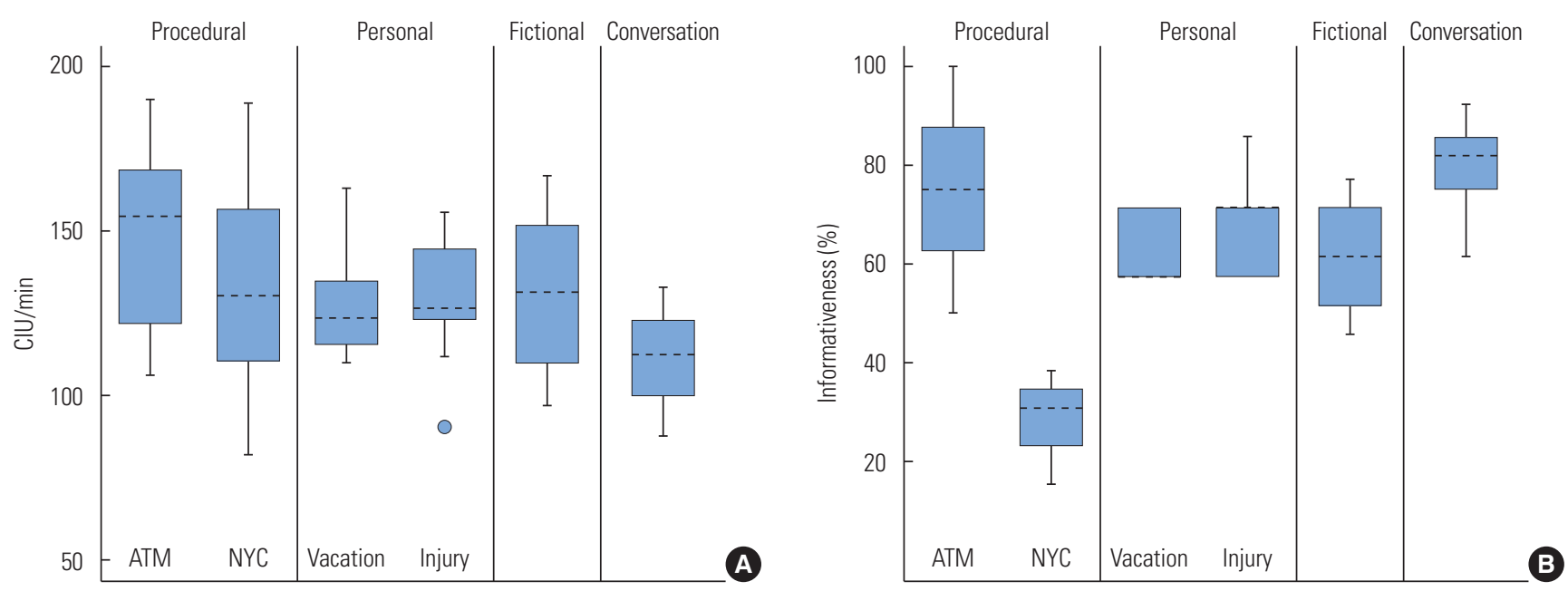

Figure 2. (A) Discourse efficiency by genre. Procedural discourse is represented by two tasks: the ATM task and the NY City task. Personal narratives are represented by the Vacation task and Injury task. Median indicated by dashed lines. (B) Discourse informativeness by genre. Median indicated by dashed lines.

correction yielding type II error in preliminary research designs. The six conditions (i.e., six different measures of the four genres) were compared two at a time resulting in 15 paired comparisons. For discourse efficiency there were no statistically significant differences between the four genres $\left(F_{(5,5)}=2.60, p=0.159\right)$. There were significant differences in the informativeness of discourse between the genres $\left(F_{(5,5)}=\right.$ $56.57, p<0.001)$. There were nine significant paired differences: between ATM and NYC $t(9)=8.07, p<0.001$; between ATM and Vaca, $t(9)=2.93, p=0.017$; between NYC and Vaca, $t(9)=-9.49, p<0.001$; between NYC and Injury, $t(9)=-9.55, p<$ 0.001; between NYC and Fict, $t(9)=-8.33, p<0.001$; between NYC and Conv, $t(9)=-17.30, p<0.001$; between Vaca and Conv, $t(9)=-6.40, p<0.001$; between Injury and Conv, $t(9)=-3.66$, $p=0.005$; and between Fict and Conv, $t(9)=-3.65, p=0.005$. Medians and variance for the efficiency measure are presented in boxplots in Figure 2A, and for informativeness in Figure 2B.

Task-related neurovascular activation in the PFC was de- rived from significant $\beta$-weights from the 16 optode receptors for each participant, and aggregated by PFC region of interest (ROI): left lateral, left medial, right medial, and right lateral $\mathrm{PFC}$, each ROI receiving data from four optodes. Significant $\beta$-weights from each of the four ROIs were summed, for each of the four discourse genres. Differences between genres were tested using two-sample $t$-tests using variables with unequal variances (Table 3, Figure 3). There were no significant differences in cortical neurovascular activation pattern amplitudes between the four genres.

Pairwise Pearson product-moment correlations were calculated between both behavioral measures (efficiency and informativeness) and neurovascular activation (Table 4) for each of the four genres. As noted above, the NYC task was chosen to represent procedural discourse, and the Injury task was chosen to represent personal narratives. There was a significant negative correlation between behavioral performance in Fictional narrative and neurovascular activation (Figure 4). 
Table 3. Paired 2-sample $t$ tests of neurovascular activation by genre

\begin{tabular}{lcccccccc}
\hline & \multicolumn{2}{c}{ Proc_NYC } & & \multicolumn{2}{c}{ Inj } & & \multicolumn{2}{c}{ Fict } \\
\cline { 2 - 3 } \cline { 8 - 9 } \cline { 7 - 8 } & $t$ & $p$ & & $t$ & $p$ & & $t$ & $p$ \\
\hline Pers_Inj & -1.33 & 0.203 & & - & - & & - \\
Fict & -0.50 & 0.62 & & 0.80 & 0.44 & & - & - \\
Conv & 0.26 & 0.80 & & 1.50 & 0.15 & & 0.70 & 0.49 \\
\hline
\end{tabular}

Table 4. Pearson pairwise correlations between behavioral performance (efficiency) and neurovascular activation for each discourse genre

\begin{tabular}{lcc}
\hline & $r$ & $p$ \\
\hline Proc_NYC & -0.071 & 0.868 \\
Pers_Inj & -0.379 & 0.355 \\
Fict & -0.782 & $0.038^{*}$ \\
Conv & 0.002 & 0.996
\end{tabular}

*Significant at $p \leq 0.05$.

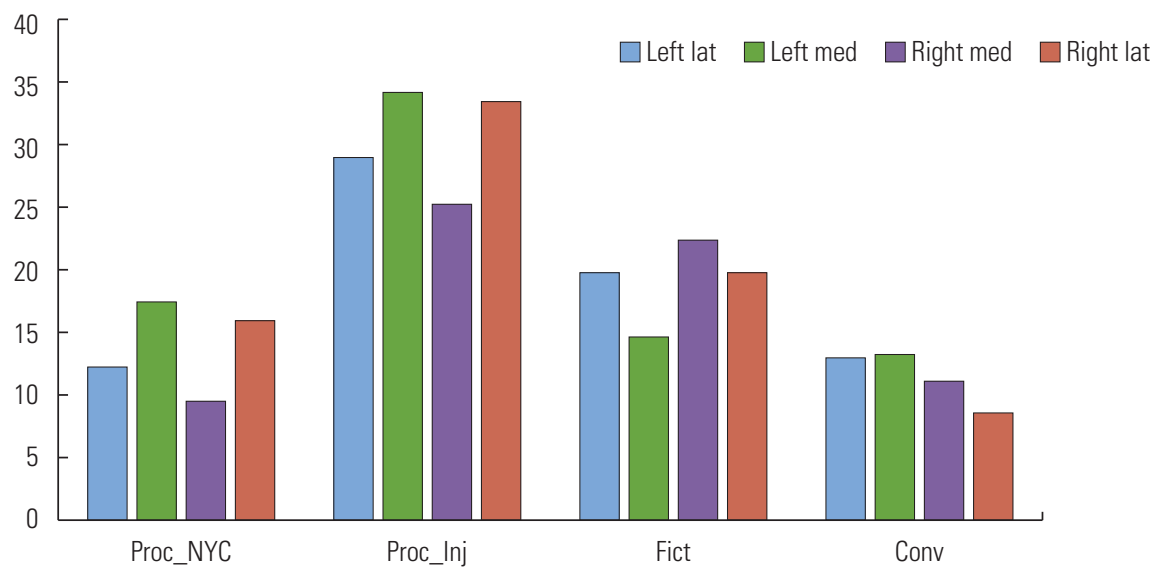

Figure 3. Aggregate neurovascular activation across all participants during formulation of each discourse genre, with activation reported by region of interest (ROI) in the PFC. For each genre, blue bar is left lateral activation, green bar is left medial activation, purple bar is right medial activation, and red bar is right lateral activation. Y-axis units are sum of significant $\beta$-weights for the four optodes per ROI, higher amplitude represents greater neurovascular activation.

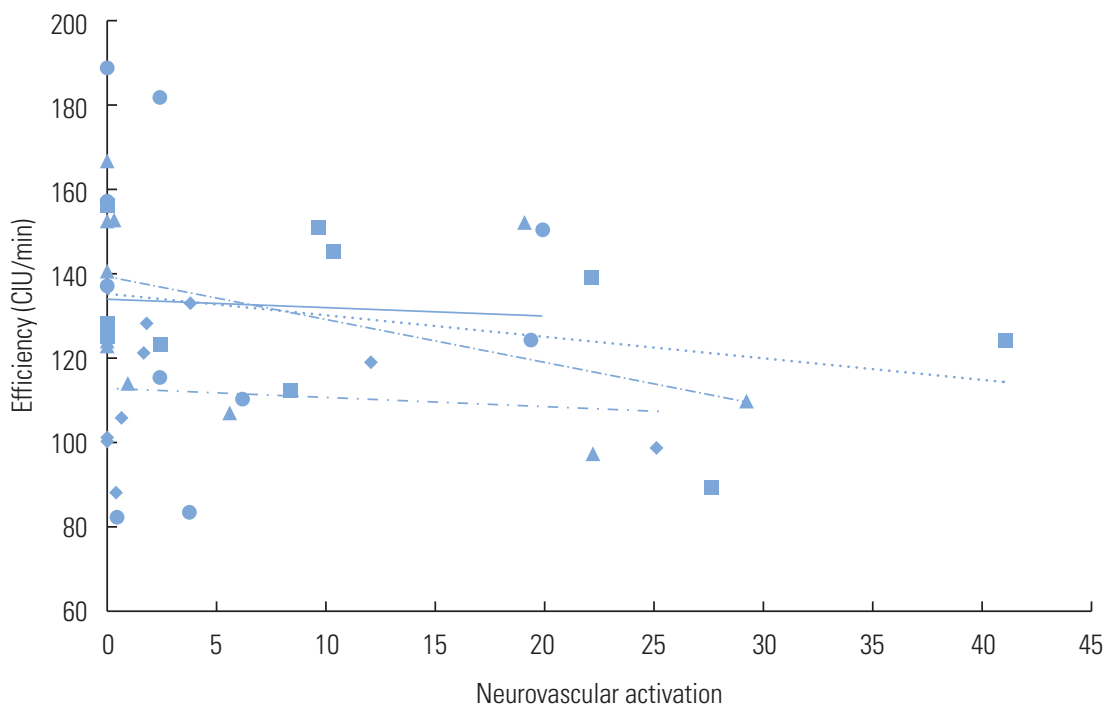

Figure 4. Correlation between Efficiency measures of behavioral performance ( $Y$ axis) and neurovascular activation ( $X$ axis; aggregate significant $\beta$-weights per participant) for each discourse genre, with linear trendlines. Circles \& solid line=Procedural_NYC; Squares \& dotted line=Personal_Injury; Triangles \& dash line =Fiction; Diamonds $\&$ dash-dot=Conversation .

\section{DISCUSSION}

The expression of human discourse is highly diverse, and the frequency, complexity, and salience of different genres is highly variable, depending on the individual and the social and functional context. However, there are certain genres of 
discourse that are critically important in vocational, social, and other familiar contexts. Past research has primarily focused on production of short, simple fictional narratives (often 5 sentences or fewer), despite the low frequency and weak functional relevance of that type of discourse in common vocational and social contexts. The genre of fictional narratives alone may be too coarse a measure to sensitively and reliably gauge impairment in discourse. We attempted to ascertain if one microlinguistic measure (efficiency) and one macrolinguistic measure (informativeness) would facilitate the analysis of more ecologically relevant discourse genres, and whether the study of one or two genres (as opposed to four or more) would yield sufficient functional and/or clinical information about an individual's discourse formulation. Hypothesis one was supported: there was no difference between the efficiency of the four genres. Given this finding, it may be valid when studying discourse efficiency to measure only the most ecologically relevant discourse genres. Hypothesis two was also supported, that there were significant differences in informativeness between the genres. Among procedural discourse tasks, the more complex NYC task likely would have higher social and vocational relevance than the ATM task, and it evoked slightly lower efficiency and significantly lower informativeness. It is reasonable to expect that discourse tasks with the highest complexity and lowest behavioral performance characteristics would be most susceptible to impairment with brain injury.

There were no significant differences in neurovascular activation between the genres. This is likely due to gaps in the neuroimaging signal caused when the activation threshold falls below the established $\beta$-weight cutoff; this had the effect of increasing the variance in the signal, and larger sample sizes will be needed to overcome this high degree of variability. It is worth noting that there appears to be a nonsignificant trend toward higher activation in the Personal injury genre. If that is the case, it may be related to the emotional salience of stories of personal injury, or because of the complexity of representing an injury to an unfamiliar listener. Research on the neural underpinnings of autobiographical memory have noted that the medial PFC is central to a functionally connected network of brain regions that support cognitive processes involved in 'self-projection': autobiographical memory, prospection (imagining the future), spatial navigation, and theory of mind [62].

There were no significant correlations between behavior (Efficiency) and neurovascular activation in any of the genres.
There was a nonsignificant trend for increases in efficiency to be associated with decreases in neurovascular activation in personal and fiction narratives. This seemingly counterintuitive observation of better performance with lower mental effort has been extensively reported in studies of physical movement efficiency, and as noted above, in discourse formulation where adherence to a predictable narrative structure lowers task-related neurovascular activation. It is plausible to consider that greater efficiency in particular (as opposed to other microstructural measures such as productivity or grammaticality) may in fact be evoked with lower neurovascular activation, and that in individuals with TBI the opposite pattern may be expected: higher activation resulting in lower performance.

This study was limited by its relatively small sample size, as well as by the high variability in both behavioral measures and neurovascular activation. This degree of variability in the fNIRS signal in particular, calculating only significant $\beta$-weights/having a cutoff and therefore not a continuous variable, prevented a full utilization of its spatial resolution, and necessitated aggregating data into four regions of interest, therefore limiting the specificity of the brain activation data. Future research will observe discourse behavior and neurovascular activation among individuals with TBI. Larger sample sizes will likely resolve these issues, but relatively high variability in both discourse formulation and in neurovascular activation is likely to remain the rule. This may require use of single case research designs, and such designs also work well for intervention research where there is a high degree of individual tailoring of therapy to the individual [63].

Recent research has noted that differences in performance between sentence-based tasks versus discourse tasks appear to be the result of variation in the executive processes that support microstructural processing, such as attention, planning, monitoring, perspective-taking, and incorporating pragmatics or context into discourse [19]. The same researchers also noted deficits in timing (more pauses and mazes) within sentences, and errors of cohesion between sentences in individuals with TBI but without aphasia, leading to the plausible proposal that microstructural and macrostructural processes are not independent, but share foundational cognitive resources. The discourse of individuals with TBI will likely contain both microstructural deficits in speed or efficiency, and macrostructural deficits in cohesion, global coherence, and/ or informativeness. It is important to continue to refine measurement and treatment of both types of deficit in ecologically 
relevant genres of discourse.

\section{ACKNOWLEDGMENTS}

The authors thank Alan Howard for statistical assistance and Ani Harlan for assistance with analyzing the data.

\section{REFERENCES}

1. Coelho C, Lê K, Mozeiko J, Krueger F, Grafman J. Discourse production following injury to the dorsolateral prefrontal cortex. Neuropsychologia. 2012;50:3564-3572.

2. van Dijk TAV, editor. Handbook of Discourse Analysis: Disciplines of Discourse. London ; Orlando: Academic Press; 1985. p. 302.

3. Coelho CA. Management of discourse deficits following traumatic brain injury: progress, caveats, and needs. Semin Speech Lang. 2007;28:122-135.

4. Lê K, Coelho C, Mozeiko J, Grafman J. Measuring goodness of story narratives. J Speech Lang Hear Res JSLHR. 2011;54:118-126.

5. Meulenbroek P, Turkstra LS. Job stability in skilled work and communication ability after moderate-severe traumatic brain injury. Disabil Rehabil. 2016;38:452-461.

6. World Health Organization. International Classification of Functioning, Disability and Health: Short Version: ICF. Geneva: World Health Organization; 2001.

7. Body R, Perkins MR. Validation of linguistic analyses in narrative discourse after traumatic brain injury. Brain Inj. 2004;18:707-724.

8. Cannizzaro MS, Coelho CA. Analysis of narrative discourse structure as an ecologically relevant measure of executive function in adults. J Psycholinguist Res. 2013;42:527-549.

9. Coelho CA. Discourse production deficits following traumatic brain injury: a critical review of the recent literature. Aphasiology. 1995;9:409-429.

10. Coelho CA, Grela B, Corso M, Gamble A, Feinn R. Microlinguistic deficits in the narrative discourse of adults with traumatic brain injury. Brain Inj. 2005;19:1139-1145.

11. Koutsoftas $\mathrm{AD}$, Gray S. Comparison of narrative and expository writing in students with and without language-learning disabilities. Lang Speech Hear Serv Sch. 2012;43:395-409.

12. Ash S, Moore P, Antani S, McCawley G, Work M, Grossman M. Trying to tell a tale: discourse impairments in progressive aphasia and frontotemporal dementia. Neurology. 2006;66:1405-1413.

13. Dijkstra K, Bourgeois MS, Allen RS, Burgio LD. Conversational coherence Discourse analysis of older adults with and without dementia. J Neurolinguistics. 2004;17:263-283.

14. Garcia L, Joanette Y. Conversational topic-shifting analysis in dementia. In: Bloom RL, Obler LK, De Santi S, Ehrlich J, editors. Discourse Analysis and Applications: Studies in Adult Clinical Populations. 1 edition. Hillsdale, NJ: Psychology Press; 2015.

15. Pistono A, Pariente J, Bézy C, Pastor J, Tran TM, Renard A, et al. Inter-individual variability in discourse informativeness in elderly populations. Clin Linguist Phon. 2017;31:391-408.
16. Whitworth A, Claessen M, Leitão S, Webster J. Beyond narrative: Is there an implicit structure to the way in which adults organise their discourse? Clin Linguist Phon. 2015;29:455-481.

17. Marini A, Galetto V, Zampieri E, Vorano L, Zettin M, Carlomagno S. Narrative language in traumatic brain injury. Neuropsychologia. 2011;49:2904-2910.

18. Marini A, Zettin M, Galetto V. Cognitive correlates of narrative impairment in moderate traumatic brain injury. Neuropsychologia. 2014;64:282-288.

19. Peach RK, Coelho CA. Linking inter- and intra-sentential processes for narrative production following traumatic brain injury: implications for a model of discourse processing. Neuropsychologia. 2016;80:157-164.

20. Angeleri R, Bosco FM, Zettin M, Sacco K, Colle L, Bara BG. Communicative impairment in traumatic brain injury: a complete pragmatic assessment. Brain Lang. 2008;107:229-245.

21. Galetto V, Andreetta S, Zettin M, Marini A. Patterns of impairment of narrative language in mild traumatic brain injury. J Neurolinguistics. 2013;26:649-661.

22. Sterr A, Herron KA, Hayward C, Montaldi D. Are mild head injuries as mild as we think? Neurobehavioral concomitants of chronic post-concussion syndrome. BMC Neurol. 2006;6:7.

23. Stout CE, Yorkston KM, Pimentel JI. Discourse production following mild, moderate, and severe traumatic brain injury. J Med Speech-Lang Pathol. 2000;8:15-25.

24. Galski T, Tompkins C, Johnston MV. Competence in discourse as a measure of social integration and quality of life in persons with traumatic brain injury. Brain Inj. 1998;12:769-782.

25. Ferstl EC, von Cramon DY. The role of coherence and cohesion in text comprehension: an event-related fMRI study. Brain Res Cogn Brain Res. 2001;11:325-340.

26. Justice LM, Bowles RP, Kaderavek JN, Ukrainetz TA, Eisenberg SL, Gillam RB. The index of narrative microstructure: a clinical tool for analyzing school-age children's narrative performances. Am J Speech Lang Pathol. 2006;15:177-191.

27. Marini A, Zettin M, Bencich E, Bosco FM, Galetto V. Severity effects on discourse production after TBI. J Neurolinguistics. 2017; 44:91-106.

28. Matsuoka K, Kotani I, Yamasato M. Correct information unit analysis for determining the characteristics of narrative discourse in individuals with chronic traumatic brain injury. Brain Inj. 2012;26: 1723-1730.

29. Nicholas LE, Brookshire RH. A system for quantifying the informativeness and efficiency of the connected speech of adults with aphasia. J Speech Hear Res. 1993;36:338-350.

30. Capilouto G, Wright HH, Wagovich SA. CIU and main event analyses of the structured discourse of older and younger adults. J Commun Disord. 2005;38:431-444.

31. Heilmann J, Miller JF, Nockerts A, Dunaway C. Properties of the narrative scoring scheme using narrative retells in young schoolage children. Am J Speech Lang Pathol. 2010;19:154-166.

32. Lehman Blake M. Clinical relevance of discourse characteristics after right hemisphere brain damage. Am J Speech Lang Pathol. 
2006;15:255-267.

33. Troiani V, Fernández-Seara MA, Wang Z, Detre JA, Ash S, Grossman M. Narrative speech production: an fMRI study using continuous arterial spin labeling. NeuroImage. 2008;40:932-939.

34. McCabe A, Bliss L, Barra G, Bennett M. Comparison of personal versus fictional narratives of children with language impairment. Am J Speech Lang Pathol. 2008;17:194-206.

35. Coelho C, Youse K, Le K, Feinn R. Narrative and conversational discourse of adults with closed head injuries and non-brain-injured adults: a discriminant analysis. Aphasiology. 2003;17:499510.

36. Cannizzaro MS, Stephens SR, Breidenstein M, Crovo C. Prefrontal cortical activity during discourse processing: an observational fnirs study. Top Lang Disord. 2016;36:65-79.

37. Gillam SL, Gillam RB. Narrative discourse intervention for schoolaged children with language impairment: supporting knowledge in language and literacy. Top Lang Disord. 2016;36: 20-34.

38. Erez AB-H, Rothschild E, Katz N, Tuchner M, Hartman-Maeir A. Executive functioning, awareness, and participation in daily life after mild traumatic brain injury: a preliminary study. Am J Occup Ther Off Publ Am Occup Ther Assoc. 2009;63:634-640.

39. Dikmen S, Machamer J, Temkin N. Mild traumatic brain injury: longitudinal study of cognition, functional status, and post-traumatic symptoms. J Neurotrauma. 2017;34:1524-1530.

40. Qian T, Jaeger TF. Cue effectiveness in communicatively efficient discourse production. Cogn Sci. 2012;36:1312-1336.

41. Bishop DVM. Research Review: Emanuel Miller Memorial Lecture 2012 - neuroscientific studies of intervention for language impairment in children: interpretive and methodological problems. J Child Psychol Psychiatry. 2013;54:247-259.

42. Mason R, Just M. Identifying component discourse processes from their fMRI time course signatures. In: Britt A, Goldman S, Rouet J-F, editors. Reading - From Words to Multiple Texts. 1 edition. New York, NY: Routledge; 2012.

43. Mason R, Just M. Neuroimaging contributions to the understanding of discourse processes. In: Traxler M, Gernsbacher MA, editors. Handbook of Psycholinguistics. 2 edition. Boston: Academic Press; 2006.

44. Ferstl EC, Neumann J, Bogler C, von Cramon DY. The extended language network: a meta-analysis of neuroimaging studies on text comprehension. Hum Brain Mapp. 2008;29:581-593.

45. Xu J, Kemeny S, Park G, Frattali C, Braun A. Language in context: emergent features of word, sentence, and narrative comprehension. NeuroImage. 2005;25:1002-1015.

46. Cannizzaro MS, Dumas J, Prelock P, Newhouse P. Organizational structure reduces processing load in the prefrontal cortex during discourse processing of written text: implications for high-level reading issues after TBI. Perspect Neurophysiol Neurogenic Speech Lang Disord. 2012;22:67-78.

47. AbdulSabur NY, Xu Y, Liu S, Chow HM, Baxter M, Carson J, et al. Neural correlates and network connectivity underlying narrative production and comprehension: a combined fMRI and PET study. Cortex J Devoted Study Nerv Syst Behav. 2014;57:107-127.

48. Quaresima V, Bisconti S, Ferrari M. A brief review on the use of functional near-infrared spectroscopy (fNIRS) for language imaging studies in human newborns and adults. Brain Lang. 2012;121: 79-89.

49. Szekely A, Jacobsen T, D’Amico S, Devescovi A, Andonova E, Herron $\mathrm{D}$, et al. A new on-line resource for psycholinguistic studies. J Mem Lang. 2004;51:247-250.

50. Snow P, Douglas J, Ponsford J. Procedural discourse following traumatic brain injury. Aphasiology. 1997;11:947-967.

51. Fleming VB, Harris JL. Complex discourse production in mild cognitive impairment: detecting subtle changes. Aphasiology. 2008;22:729-740.

52. Peterson C. Developmental psycholinguistics: three ways of looking at a child's narrative. 1 edition. New York: Springer; 1983. p. 245.

53. Berman RA, Slobin DI, editors. Relating events in narrative: a crosslinguistic developmental study. 1 edition. London; New York: Psychology Press; 2016. p. 764.

54. Damico J. Clinical Discourse Analysis: A functional approach to language assessment. In: Simon CS, editor. Communication Skills and Classroom Success: Assessment and Therapy Methodologies for Language and Learning Disabled Students. Eau Claire, WI: Thinking Pubns; 1991.

55. Grice H. Logic and Conversation. In: Cole P, Morgan JL, editors. Syntax and Semantics, Volume 3: Speech Acts. New York, NY: Academic Press; 1975. p. 41-58.

56. Hunt KW. Grammatical structures written at three grade levels. NCTE Research report No. 3. Champlain, IL: National Council of Teachers of English; 1965. p. 159.

57. Ayaz H, Izzetoglu M, Platek SM, Bunce S, Izzetoglu K, Pourrezaei K, et al. Registering fNIR data to brain surface image using MRI templates. Conf Proc Annu Int Conf IEEE Eng Med Biol Soc IEEE Eng Med Biol Soc Annu Conf. 2006;1:2671-2674.

58. fNIR Devices LLC. fNIR Imager \& COBI Studio Manual [Internet]. Potomac, MD: fNIR Devices LLC; 2018. Available from: https:// www.biopac.com/wp-content/uploads/fnir-cobi-manual.pdf

59. Ye JC, Tak S, Jang KE, Jung J, Jang J. NIRS-SPM: statistical parametric mapping for near-infrared spectroscopy. NeuroImage. 2009;44: 428-447.

60. Pfeifer MD, Scholkmann F, Labruyère R. Signal processing in functional near-infrared spectroscopy (fNIRS): methodological differences lead to different statistical results. Front Hum Neurosci. 2017;11:641.

61. Jang KE, Tak S, Jung J, Jang J, Jeong Y, Ye JC. Wavelet minimum description length detrending for near-infrared spectroscopy. J Biomed Opt. 2009;14:034004.

62. Spreng RN, Mar RA, Kim ASN. The common neural basis of autobiographical memory, prospection, navigation, theory of mind, and the default mode: a quantitative meta-analysis. J Cogn Neurosci. 2009;21:489-510.

63. Shadish WR. Analysis and meta-analysis of single-case designs: an introduction. J Sch Psychol. 2014;52:109-122. 